\title{
Red Yeast Rice Mitigates High-Fat Diet Induced-Obesity Related Vascular Dysfunction in Wistar Albino Rats
}

\author{
Hassan A Madkhali 1,* ${ }^{(\mathbb{D})}$, Majid A Ganaie ${ }^{2(\mathbb{D}}$, Mohd Nazam Ansari ${ }^{1}{ }^{\mathbb{D}}$, Khalid M Alharthy ${ }^{1}{ }^{\mathbb{D}}$, Najeeb \\ Ur Rehman ${ }^{1}{ }^{(\mathbb{D}}$, Mohammed A Alamri ${ }^{1 \mathbb{D}}$, Faisal K Alkholifi ${ }^{1}{ }^{(\mathbb{D}}$, Abubakr M Hamad ${ }^{3,4} \mathbb{D}^{\mathbb{D}}$, Abdullah \\ Hamadi 5 iD
}

1 Department of Pharmacology and Toxicology, College of Pharmacy, Prince Sattam Bin Abdulaziz University, Al-Kharj, Saudi Arabia; dr_madkhali@hotmail.com (H.A.M.), nazam.ansari@gmail.com (M.N.A.), k.alharthy@psau.edu.sa (K.M.A.), n.rehman@psau.edu.sa (N.U.R.), ma.alamri@psau.edu.sa (M.A.A.), f.alkholifi@psau.edu.sa (F.K.A.);

2 College of Dentistry and Pharmacy, Buraydah Colleges, Buraydah, Saudi Arabia; majidsays@gmail.com (M.A.A.);

3 Department of Basic Science, Preparatory year Deanship, Prince Sattam Bin Abdulaziz University, Al-Kharj, Saudi Arabia

4 Department of Histopathology and Cytopathology, Faculty of Medical Laboratory Sciences, University of Gezira, Wad Madani, Sudan; abkr.hamad@gmail.com (A.M.H.);

5 Faculty of Applied Medical Sciences, Medical Laboratory Technology, University of Tabuk, Tabuk, Saudi Arabia; a.aldhafri@ut.edu.sa (A.H.);

* Correspondence: dr_madkhali@hotmail.com (H.A.M.);

Scopus Author ID 57200310086

Received: 25.01.2021; Revised: 25.02.2021; Accepted: 3.03.2021; Published: 9.03.2021

\begin{abstract}
The red yeast rice methanolic extract (RYR) was suggested to have promising therapeutic effects against vascular endothelial dysfunction. In the present study, the protective effects of RYR at 200 and $400 \mathrm{mg} / \mathrm{kg} /$ day were investigated in a specific animal model that exhibits a high-fat diet (HFD)induced dyslipidemia and vascular endothelium dysfunction. Vascular endothelial reactivity experiments were evaluated using aorta obtained from adult rats in an ex-vivo organ bath setup. Three consecutive weeks of RYR treatment exhibited a significant reduction in body weight (BW), liver weight (LW), and retroperitoneal fat pad weight (RFPW)/BW ratios as compared to HFD only treated rats where a significant body weight gain was observed. RYR treatment also significantly decreased the average daily food intake, waist, lee index, and body mass index compared to rats treated with HFD only. RYR treatment significantly reduced total cholesterol, triglyceride, low-density lipoprotein, and very-low-density lipoprotein levels compared to the HFD group. HFD-induced endothelium dysfunction in the aorta of animals was reversed by RYR treatment, comparable to the HFD group. RYR treatment, specifically at $400 \mathrm{mg} / \mathrm{kg} / \mathrm{day}$ dose, revealed a significant protective effect on vascular endothelium and an improvement in the lipid panel, hence justifying therapeutic involvement of RYR in dyslipidemia and endothelial dysfunctions.
\end{abstract}

Keywords: red yeast rice; lipid profile; high-fat diet; endothelial dysfunction.

(C) 2021 by the authors. This article is an open-access article distributed under the terms and conditions of the Creative Commons Attribution (CC BY) license (https://creativecommons.org/licenses/by/4.0/).

\section{Introduction}

Obesity prevalence has continued to increase and is becoming an economic burden in developed and developing countries due to its link with several other comorbidities. The standard threshold for obesity is a body mass index (BMI) of $30 \mathrm{~kg} / \mathrm{m}^{2}$, and it was arranged into several classes based on this measurement of weight to the body surface. Obesity etiology includes several genetic predispositions favoring weight gain [1-4]. Environmental causes such 
as conveniences in daily life due to modernity lead to an increasingly sedentary lifestyle and high caloric intake [5-7].

Epidemiological data shows that, in general, obese populations are on the rise both globally and within the Gulf Cooperation Council (GCC) countries. For example, in the United States, obesity has been doubled among adults since 1980 [8-10]. Regional trends estimated obesity occurrence in adults was range from 13 to $50 \%$ in the Gulf countries [11]. The WHO ranked Saudi Arabia, the United Arab Emirates, and Kuwait within the top 20 highest countries in terms of obesity prevalence.

Of particular interest to our work is endothelial dysfunction, which occurs during obesity and is an underlying mechanism of cardiovascular disorders such as hypertension, coronary artery disease, and atherosclerosis $[12,13]$. This is because endothelial dysfunction reduces the availability of nitric oxide (NO) and, thus, the ability of vasculature to dilate $[14,15]$. A High-fat diet was found to cause obesity and inflammatory oxidative stress markers in several experiments [16-19]. Hence, endothelial dysfunction may be an excellent target to reverse or prevent the complications of obesity and HFD due to its link with the events mentioned above, such as oxidative stress.

While most clinically use medications are of synthetic origin, natural compounds and extracts can still exhibit promising therapeutic effects [20]. For example, many natural origin compounds showed an ability to reduce obesity and dyslipidemia biomarkers [21,22]. In this research, we pay attention to red yeast rice (RYR), which is the rice that has been fermented using Monoscus purpureus mold [23]. Meta-analysis and randomized trials using RYR have repeatedly shown significant beneficial health effects such as reducing total serum cholesterol (TC), low-density lipoprotein (LDL), and triglycerides (TG) [24-27]. RYR was shown to be composed of various molecules pertaining to sterols, unsaturated fatty acids, flavones, and most importantly, monacolin K $[28,29]$. The latter of which (better known as lovastatin) was found to be an inhibitor of 3-hydroxy-3-methylglutaryl-CoA (HMG CoA) reductase, resulting in reduced cholesterol synthesis and levels in the body [30].

While the activity of RYR against cholesterol and triglyceride levels is wellestablished, its other beneficial effects are less explored. Furthermore, the therapeutic role of RYR in an animal model of dyslipidemia-mediated endothelial dysfunction has not been reported. Therefore, in the current investigation, we assess the possible therapeutic involvement of RYR on HFD-fed obese rats which exhibited endothelial dysfunction.

\section{Materials and Methods}

\subsection{Chemicals and reagents.}

Acetylcholine chloride (a cholinergic agonist), phenylephrine (adrenergic agonist), and kits for the estimation of total cholesterol, triglyceride, and high-density lipoprotein (HDL) in serum were procured from Sigma Aldrich Chemical Co (USA). The chemicals used to make Krebs solution were purchased from E. Merck KGaA (Darmstadt, Germany). All the drugs and extract were prepared as stock, and fresh dilutions were prepared before the relevant experiments. 


\subsection{Animals.}

Healthy male and female Wistar albino rats (150-180 g) were picked up from the Animal care Unit of College of Pharmacy, Prince Sattam Bin Abdulaziz University (PSAU), Kingdom of Saudi Arabia (KSA). The study protocol was approved by the Institutional Animal Ethics Committee, College of Pharmacy, PSAU, KSA. During the acclimation period of one week and during the study, animals were maintained under standard laboratory circumstances, at $25 \pm 2{ }^{\circ} \mathrm{C}$, under a $12 \mathrm{~h}$ light/12 h dark cycle, with food and water ad libitum.

\subsection{Plant extract preparation.}

Red yeast rice raw material (brand: Liew Treatment Centre; batch number: 160000152) was purchased from Kuala Lumpur, Malaysia. The dried red yeast rice powder (500 g) was submitted to extraction with methanol $(1.5 \mathrm{~L})$ by maceration at room temperature $\left(25-27^{\circ} \mathrm{C}\right)$ for 2 days. Following filtration, the extract was evaporated using a rotary vacuum evaporator until dryness under reduced pressure. The extract $(37.5 \mathrm{~g})$ was then stored in the refrigerator $\left(2-4^{\circ} \mathrm{C}\right)$ for further use, and required concentrations were prepared immediately before use.

\subsection{Preparation of high-fat diet.}

The method of Xu et al. [31] was followed with small adjustments. Briefly, a regular pellet diet, i.e., standard diet (SD), which makes up $73 \%$ of the total diet content, was pulverized and combined with the ingredients presented in Table 1. A sufficient amount of water was added to the finals blend to make semisolid pellets, which were then appropriately dried in the oven to prevent fungal contamination.

Table 1. Composition of high-fat diet (HFD).

\begin{tabular}{l|l} 
Ingredients & Percentage of HFD \\
\hline Powdered Normal Pellet Diet (NPD) & $73 \%$ \\
\hline Cholesterol & $1 \%$ \\
\hline Tallow & $10 \%$ \\
\hline Egg yolk powder & $10 \%$ \\
\hline Milk powder & $6 \%$
\end{tabular}

\subsection{Experimental design.}

Rats were randomly distributed into four groups (6 animals in each). Group I rats fed with a standard diet (SD). Group II rats fed with a high-fat diet (HFD). Group III and IV rats were co-administered with HFD and RYR at doses of 200 and $400 \mathrm{mg} / \mathrm{kg} / \mathrm{day}$ (orally), respectively (Figure 1).

\begin{tabular}{|c|c|c|c|}
\hline \multicolumn{3}{|c|}{ SD } & \multirow{2}{*}{$\begin{array}{l}\text { Control group } \\
\text { Vehicle group }\end{array}$} \\
\hline SD & \multicolumn{2}{|c|}{ HFD } & \\
\hline SD & HFD & HFD + 200 RYR & \multirow{2}{*}{$\begin{array}{l}\text { RYR groups } \\
\text { (mg/kg/day) }\end{array}$} \\
\hline SD & HFD & $\mathrm{HFD}+400 \mathrm{RYR}$ & \\
\hline
\end{tabular}

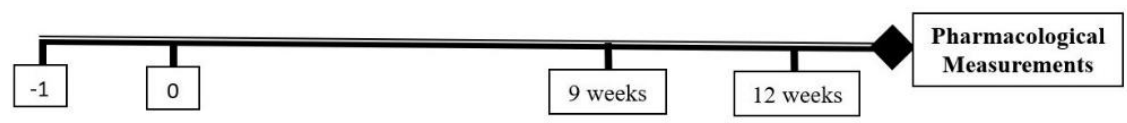

Figure 1. Schematic diagram showing the experimental design of the present study. 
2.6. Evaluation of RYR extract effects on animal $B W, L W$, and $R F P W$.

To measure the effect of RYR on animal BW, LW, and RFPW, RYR ranged doses (200 and $400 \mathrm{mg} / \mathrm{kg} / \mathrm{day}$ ) were administered to HFD fed rats orally for three weeks (rats were fed HFD for nine weeks preceding RYR administration).

\subsection{Effect of RYR on food intake.}

Each animal's total food consumption was assessed daily following the method previously reported by Diniz et al. [32]. The daily food pellets intake was determined by measuring the amount of food pellets put into the food tray and the amount left in the tray.

\subsection{Effect of RYR on different anthropometric indices.}

Several anthropometric indices, including BMI, waist circumference, and Lee index of all the rats, were evaluated by the method described earlier [33].

\subsection{Effect of RYR on lipid panel.}

By penetrating the retro-orbital sinus with a sterile capillary tube, blood samples of all animals were drawn and collected under light ether anesthesia $(0.08 \mathrm{ml} / \mathrm{L}$ of the volume of a container) in blood collection tubes and then centrifuged $\left(3,000 \mathrm{rpm}, 4^{\circ} \mathrm{C}, 20 \mathrm{~min}\right)$ for serum isolation. The isolated serum samples were kept in the refrigerator to be used for lipid analysis, including TC, TG, and HDL levels. VLDL and LDL levels were measured directly using a well-known principle [34]:

$$
\begin{gathered}
\text { VLDL }=\mathrm{TGs} / 5 \\
\mathrm{LDL}=\mathrm{TC}-(\mathrm{VLDL}+\mathrm{HDL})
\end{gathered}
$$

\subsection{Assessment of endothelial dysfunction.}

After food deprivation for 16 hours, all animals were killed with a rapid and strong blow to the head. The aortae were carefully isolated, cleaned, transversely cut to obtain rings of 2-3 mm length, and mount in an organ bath contained aerated physiological Krebs solution (37 degrees Celsius), attached to a force sensor connected with data acquisition system (emkaBath2) and IOX2 software for accurate measurement of the tension to study endothelial reactivity. Some changes followed the previously published method of Furchgott and Zawadski [35]. After mounting, the aortic tissues were stabilized for 45-60 minutes by maintaining a resting force of $2 \mathrm{~g}$ and changing the Krebs solution every 15 minutes. Following the equilibrium, contractions were induced using the submaximal concentration of alpha-1 adrenergic agonist; phenylephrine $\left(\mathrm{PE} ; 1 \times 10^{-6} \mathrm{M}\right)$. Soon after achieving sustained contractions (usually 15-20 min), Ach $\left(1 \times 10^{-9}-1 \times 10^{-5} \mathrm{M}\right)$ was added cumulatively to obtain the inhibitory concentration-response curves (CRCs).

\subsection{Aorta histological examination.}

A small part of the isolated thoracic aortae was fixed in formalin solution and further processed for paraffin embedding. A rotary microtome was used to get aortae tissue sections of $5 \mu \mathrm{m}$ thickness for histological examinations. The general histological structure, collagen fibers, and elastic fibers of all isolated aortae from rats were examined using various staining 
techniques, including hematoxylin and eosin (H\&E), van Gieson, and Verhöeff's. Following staining procedures, tissue slices were studied under a light microscope (Hund Wetzlar H600/12, Germany) connected to a digital camera.

\subsection{Statistical analysis.}

All observed data were presented as mean \pm standard error of the mean (SEM) and considered statistically significant with a $P$-value of $<0.05$. Results were evaluated using ANOVA followed by Dunnett's t-test using GraphPad Prism 7.0 (USA).

\section{Results and Discussion}

\subsection{Determination of RYR effect on body and organ weight.}

Animals with SD were in the range of $200 \mathrm{~g}$, and it was dramatically and significantly increased by up to $420 \mathrm{~g}$ when the food was replaced with HFD.
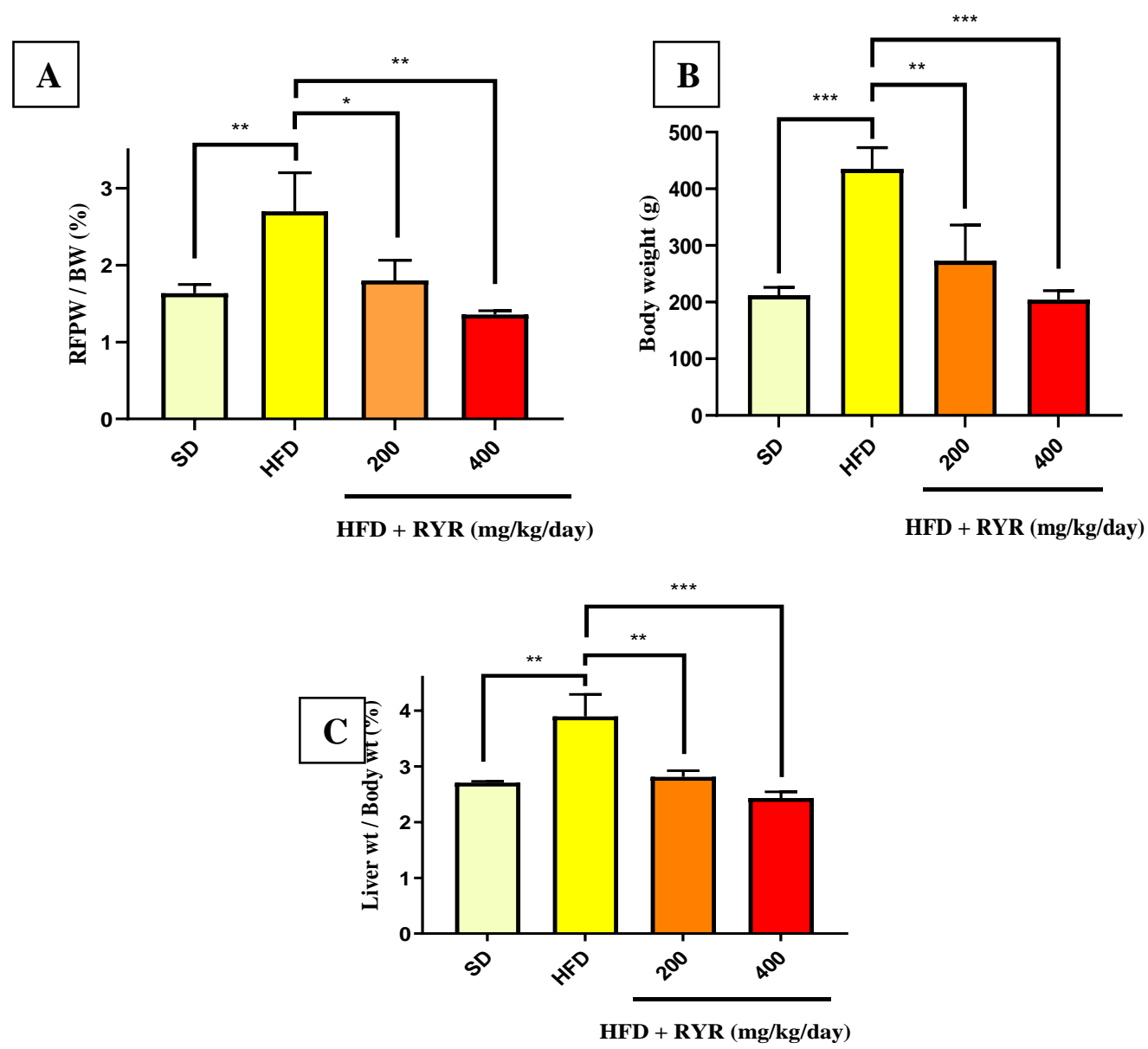

Figure 2. The change in the (A) body weight, (B) liver weight/body weight ratio, (C) retroperitoneal fat pad weight (RFPW)/body weight ratio of the experimental animals. Values represented by the mean \pm SD $(n=6), * P$ $\leq 0.05, * * \mathrm{P} \leq 0.01, * * * \mathrm{P} \leq 0.001$, represents comparison of HFD with SD (Unpaired $\mathrm{t}$-test) and treated groups (RYR; 200 and $400 \mathrm{mg} / \mathrm{kg}$ ) with HFD group.

Upon RYR treatment using 200 or $400 \mathrm{mg} / \mathrm{kg} / \mathrm{day}$ along with HFD, the mean of animals' weight was around 280 and 200 gm, respectively (Figure 2A).

Animals with SD had a liver weight that is around $2.75 \%$, and it was significantly increased by up to $3.9 \%$ when the food was replaced with HFD. However, in the animals given 200 or $400 \mathrm{mg} / \mathrm{kg} / \mathrm{day}$, the liver weight was around 3.95 and $2.4 \%$, respectively (Figure $2 \mathrm{~B}$ ). 
Each bar represents the mean for each group. Animals with SD were having RFPW, which is around $1.6 \%$. It was dramatically and significantly increased by up to $4.6 \%$ when the food was replaced with HFD. However, in the animals given 200 or $400 \mathrm{mg} / \mathrm{kg} / \mathrm{day}$, the RFPW levels were 1.8 and $1.4 \%$, respectively (Figure 2C).

\subsection{Evaluation of RYR effect on food intake.}

Food pellets intake was assessed by measuring the amount of food pellets placed into the food tray and subtracting the amount left in the tray. Statistically, no significant difference was reported between the average daily food intakes for treatment groups, with an average ranging around $20 \mathrm{~g}$ per day (Figure 3).

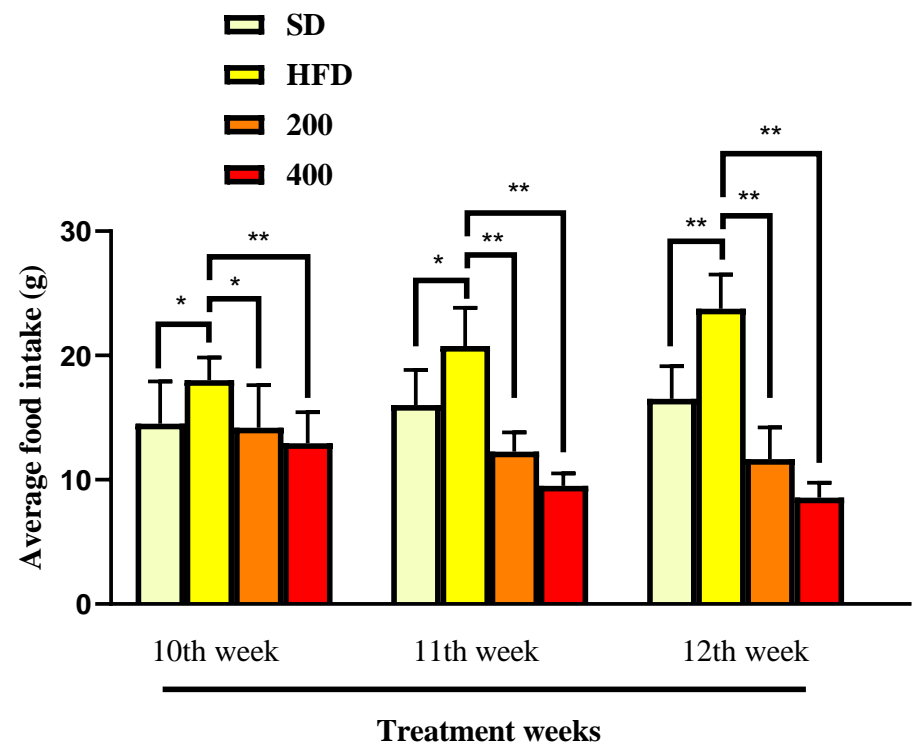

Figure 3. The average daily feed intake of all the animals. Values represented by the mean $\pm \mathrm{SD}(\mathrm{n}=6),{ }^{*} \mathrm{P} \leq$ $0.05, * * \mathrm{P} \leq 0.01$, represent a comparison of HFD with SD (Unpaired t-test) and treated groups (RYR; 200 and $400 \mathrm{mg} / \mathrm{kg}$ ) with HFD group.

\subsection{Determination of RYR effect on waist, Lee index, and BMI.}

The waist size was measured in centimeters to verify the validity of the obesity model used (Figure 4A). For the SD animals, the waist size was in the range of $15 \mathrm{~cm}$. Upon HFD feeding, animals experienced an increase in the waist for up to $20 \mathrm{~cm}$. However, when the animals received 200 or $400 \mathrm{mg} / \mathrm{kg} /$ day of RYR and HFD, they have shown a decrease in the waist size ranging in 16 and $14 \mathrm{~cm}$, respectively, compared to the HFD animals without any RYR treatment.

The same effect was seen when Lee's index and body mass index (BMI) were evaluated (Figure 5A and 5B). Lee index for the SD animals was around $0.28 \mathrm{~g} / \mathrm{cm}$, which significantly increased to 0.36 upon receiving HFD.

When the animals received 200 or $400 \mathrm{mg}$ of RYR along with HFD, Lee index was attenuated to the baseline levels seen in the SD animals in the range of $0.28 \mathrm{~g} / \mathrm{cm}$ for both treatments. However, no statistical differences between the two treatments favor any concentration for its positive effects. 


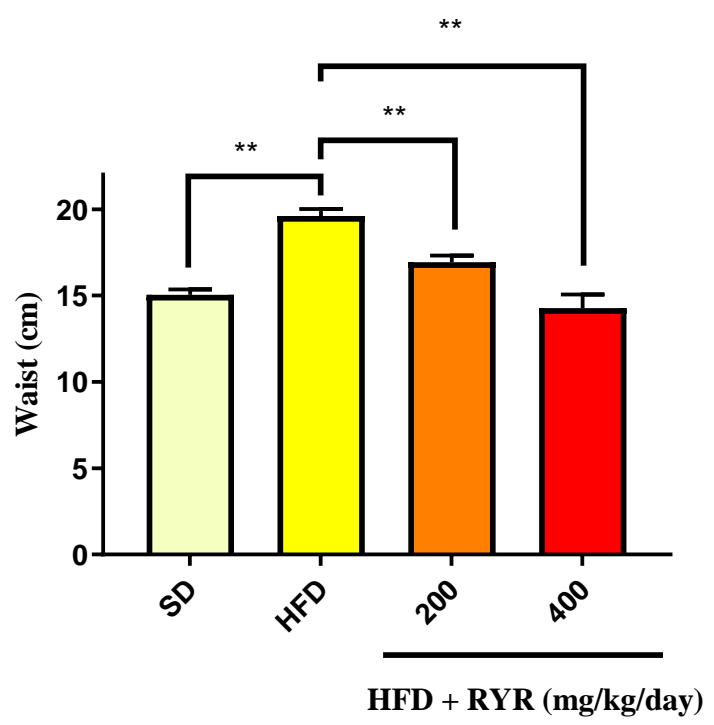

Figure 4. Effect of RYR treatment on waist size in High fat diet-induced obese rats. Values represented by the mean $\pm \mathrm{SD}(\mathrm{n}=6), * * \mathrm{P} \leq 0.01$, represent a comparison of HFD with SD (Unpaired t-test) and treated groups (RYR; 200 and $400 \mathrm{mg} / \mathrm{kg}$ ) with HFD group.

For the BMI (Figure 5B), SD animals had $4.4 \mathrm{~g} / \mathrm{cm}^{2}$ that increased up to $6.5 \mathrm{~g} / \mathrm{cm}^{2}$ upon receiving HFD. When the 200 and $400 \mathrm{mg} / \mathrm{kg} /$ day of RYR was given to animals along with $\mathrm{HFD}$, the BMI was reduced to $4.5 \mathrm{~g} / \mathrm{cm}^{2}$ and $4.1 \mathrm{~g} / \mathrm{cm}^{2}$, respectively.
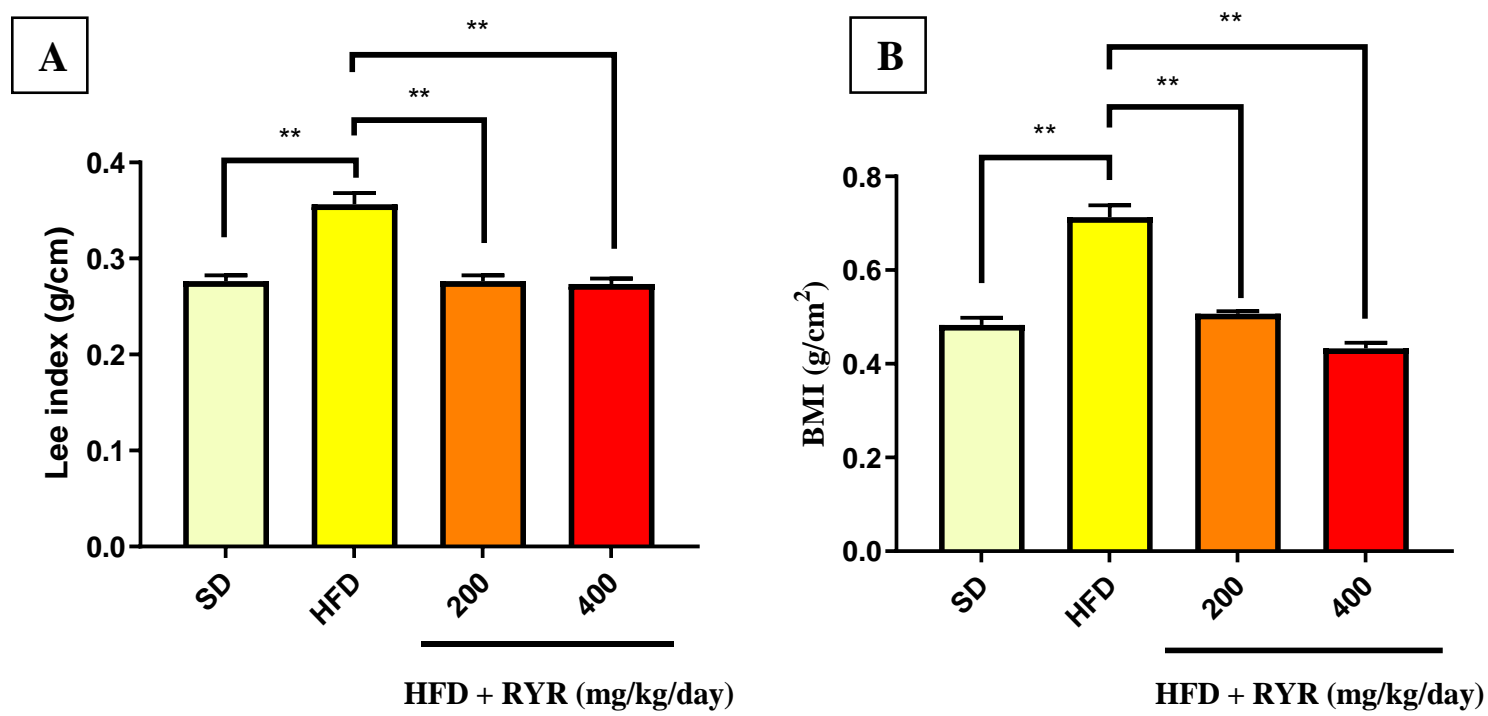

Figure 5. Effect of RYR treatment on (A) Lee index; and (B) Body mass index (BMI) on High fat diet-induced obese rats. Values represented by the mean $\pm \mathrm{SD}(\mathrm{n}=6), * * \mathrm{P} \leq 0.01$, represent a comparison of HFD with SD

(Unpaired t-test) and treated groups (RYR; 200 and $400 \mathrm{mg} / \mathrm{kg}$ ) with HFD group.

To visually explore the changes in various animal groups' fat levels, a representative image of retroperitoneal fat of various treated animal groups was shown. Animals with SD had moderate fat levels. HFD animals had an excessive amount of fats compared to the SD animals (Figure 6). 


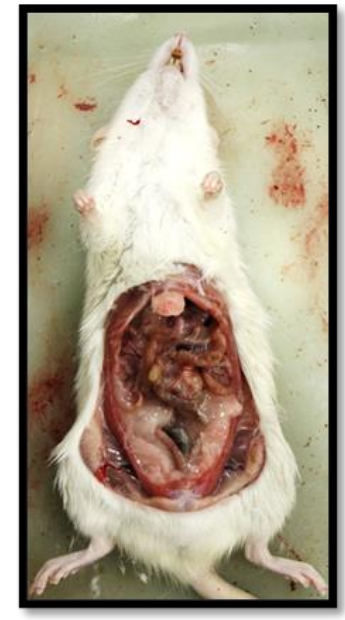

SD

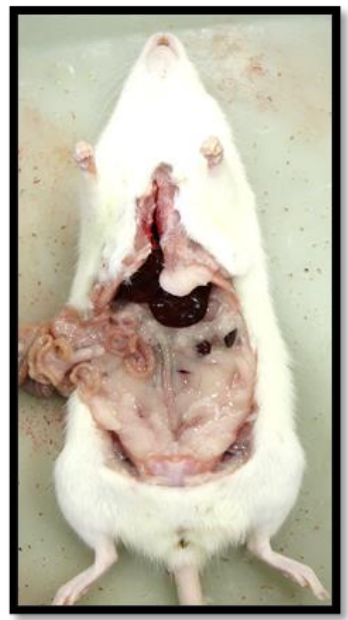

HFD

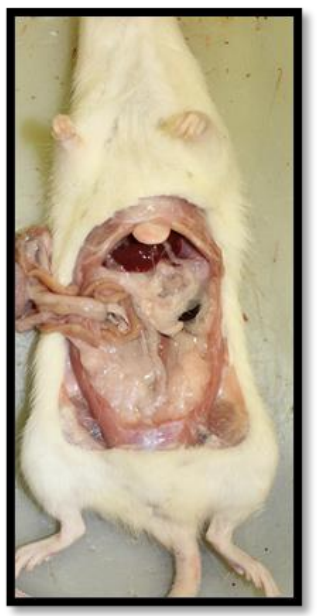

200

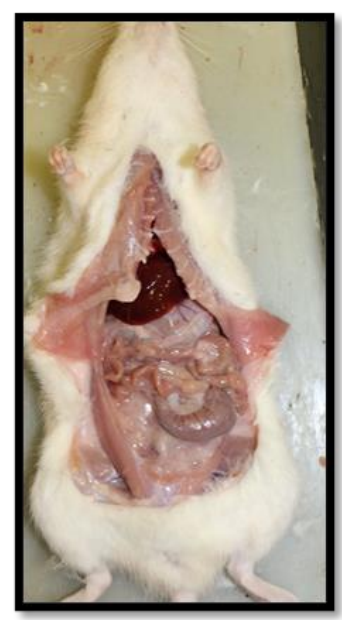

400

\section{HFD + RYR (mg/kg/day)}

Figure 6. Ventral view of dissected rats after 3 weeks treatment. Photographs of the representative of retroperitoneal fat of rats of each group; SD, HFD, HFD + RYR 200 or $400 \mathrm{mg} / \mathrm{kg} /$ day) after 3 weeks treatment (at the end of the study). SD: Standard diet; HFD: High-fat diet; RYR: Red yeast rice.

\subsection{Effect of RYR on lipid profile.}

Based on the experimental setup that has been used, animals were divided into four different treatment groups. The first group was fed SD; the second group was fed HFD; the third and fourth groups received HFD and the suggested dose of RYR of 200 or $400 \mathrm{mg} / \mathrm{kg} / \mathrm{day}$, consecutively. Various parameters were selected to be compared between treatment groups, Cholesterol, Triglycerides, and Lipoproteins in the blood. Group fed SD have shown normal levels of Cholesterol, Triglycerides, and Lipoproteins based on the range of normal levels available in the literature for this type of experiment. Groups treated with HFD exhibited a statistically significant increase in cholesterol, triglycerides, VLDL, and LDL. The only parameter that demonstrated a statistically significant decrease was HDL as compared to the SD fed group (Table 2).

Table 2. Effect of RYR on lipid profile.

\begin{tabular}{c|c|c|c|c|c} 
Groups & $\begin{array}{c}\text { Cholesterol } \\
(\mathbf{m g} / \mathbf{d L})\end{array}$ & $\begin{array}{c}\text { Triglycerides } \\
(\mathbf{m g} / \mathbf{d L})\end{array}$ & $\begin{array}{c}\text { HDL-C } \\
(\mathbf{m g} / \mathbf{d L})\end{array}$ & $\begin{array}{c}\text { VLDL-C } \\
(\mathbf{m g} / \mathbf{d L})\end{array}$ & $\begin{array}{c}\text { LDL-C } \\
(\mathbf{m g} / \mathbf{d L})\end{array}$ \\
\hline $\mathrm{SD}$ & $152.95 \pm 10.45$ & $22.91 \pm 2.72$ & $83.98 \pm 3.84$ & $4.76 \pm 0.41$ & $64.20 \pm 9.55$ \\
\hline HFD & $375.26 \pm 16.30^{* * *}$ & $85.41 \pm 5.99^{* * *}$ & $47.91 \pm 1.28^{* * * *}$ & $17.08 \pm 1.19^{* * * *}$ & $310.27 \pm 17.46^{* * * *}$ \\
\hline HFD + RYR & $355.91 \pm 14.71^{\text {ns }}$ & $52.08 \pm 3.10^{* * *}$ & $57.16 \pm 1.86^{\text {ns }}$ & $10.42 \pm 0.62^{* * *}$ & $288.33 \pm 13.81^{\text {ns }}$ \\
$200 \mathrm{mg} / \mathrm{kg} /$ day & & & & & \\
\hline $\begin{array}{c}\text { HFD + RYR } \\
400 \mathrm{mg} / \mathrm{kg} / \text { day }\end{array}$ & $265.32 \pm 13.34^{* * * *}$ & $43.05 \pm 3.30^{* * * *}$ & $59.11 \pm 3.24^{\mathrm{ns}}$ & $8.61 \pm 0.66^{* * * *}$ & $197.59 \pm 15.07^{* * * *}$ \\
& & & & &
\end{tabular}

Values represented by the mean $\pm \mathrm{SD}(\mathrm{n}=6), * * * \mathrm{P} \leq 0.001$, represent a comparison of HFD with SD (Unpaired t-test) and treated groups (RYR; 200 and $400 \mathrm{mg} / \mathrm{kg}$ ) with HFD group.

Next, the RYR treated groups were investigated for the same parameters' levels. The group that received $200 \mathrm{mg}$ RYR has shown a significant reduction level of triglycerides as compared to the HFD group. However, there was also a significant margin in the levels between the RYR $200 \mathrm{mg}$ treated group and the SD group. The same effect was seen in the VLDL parameter, where the animals treated with RYR $200 \mathrm{mg}$ produced a substantial reduction 
compared to the HFD group, but the reduced value was still significantly higher than the SD group. The other parameters, including cholesterol, HDL, and LDL, did not significantly change as compared to the HFD group.

Finally, the $400 \mathrm{mg}$ RYR treated group was investigated. The levels of all parameters except HDL produced a significant reduction in levels compared to the correspondent parameter levels from the HFD group. But this reduction was not sufficient to be close to the baseline normal levels seen in the SD group. HDL levels were not affected by any RYR treatment in the experiment.

\subsection{Effect of RYR on endothelial dysfunction.}

Different groups of animals were treated with SD, HFD, and HFD, along with RYR $(400 \mathrm{mg} / \mathrm{kg})$. After $16 \mathrm{~h}$ treatment, the thoracic aorta's endothelial reactivity was evaluated by determining the concentration of the vasoactive substances such as acetylcholine (Ach) in the endothelium. The more Ach concentration released, the healthier the endothelium. The normal healthy aorta, when they induced vasoconstriction by phenylephrine (PE) up to $20 \%$ increased the Ach concentration. On the other hand, the unhealthy aorta had significant low vasoconstriction up to $90 \%$ of the original aorta activity. Upon treatment of HFD with RYR, the loss of vasoconstriction seen in the diseased animals were restored, resulting in around 30 $\%$ vasoconstriction in HFD rats treated with RYR at a dose of $400 \mathrm{mg} / \mathrm{kg} / \mathrm{day}$ and showed endothelium-dependent vasodilatation, whereas RYR at low dose exhibited smaller relaxation compared to the higher dose (Figure 7).

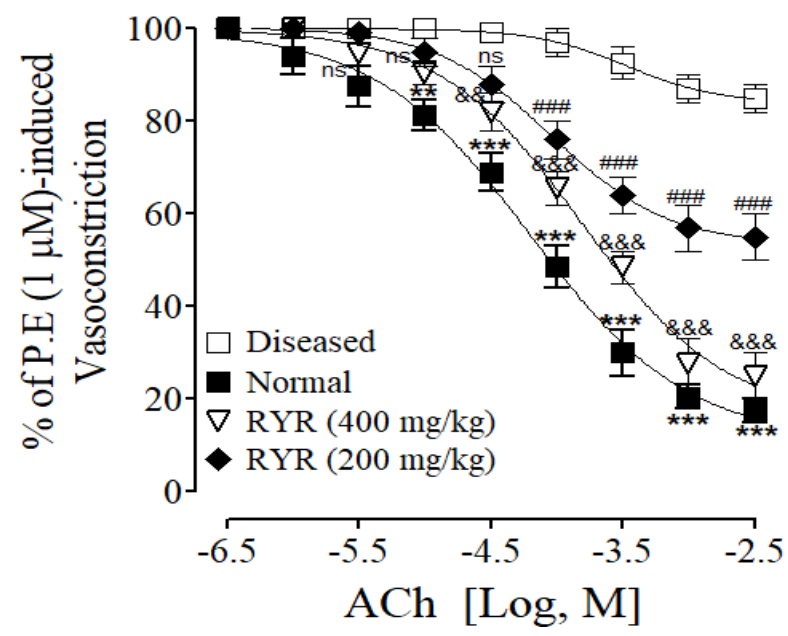

Figure 7. Effect of RYR on vascular endothelium; - SD (Normal); $\square$ HFD (Diseased); $\bullet$ HFD + RYR (200 $\mathrm{mg} / \mathrm{kg} /$ day); $\nabla$ HFD + RYR $(400 \mathrm{mg} / \mathrm{kg} /$ day). $\mathrm{ns} \mathrm{p}>0.05$, **,\#,\&\& $\mathrm{p}<0.01$ and $* * *$,\&\&\& $\mathrm{p}<0.001$ (Two-way ANOVA, followed by Bonferroni post-tests).

\subsection{Effect of RYR on histopathological changes.}

Using Hematoxylin and Eosin, histological staining of isolated rat aorta at 30x and $35 \mathrm{x}$ magnification shows that samples from rats with SD represent the ideal collagen-elastic ratio. In comparison, samples from rats with HFD have shown poor elasticity accompanied by a significant reduction in the collagen-elastic ratio. However, samples from rats with HFD treated with RYR (400 mg/kg/day) show little improvement in elastic fiber, but there was no improvement in collagen-elastic ratio. In addition, many degeneration areas are present in the area of elastic fibers (Arrow at photo 7A x30). Van Gieson and Verhoeff stains were used to 
further differentiate between collagen fibers and elastic fibers in all treatment samples (Figure 8).

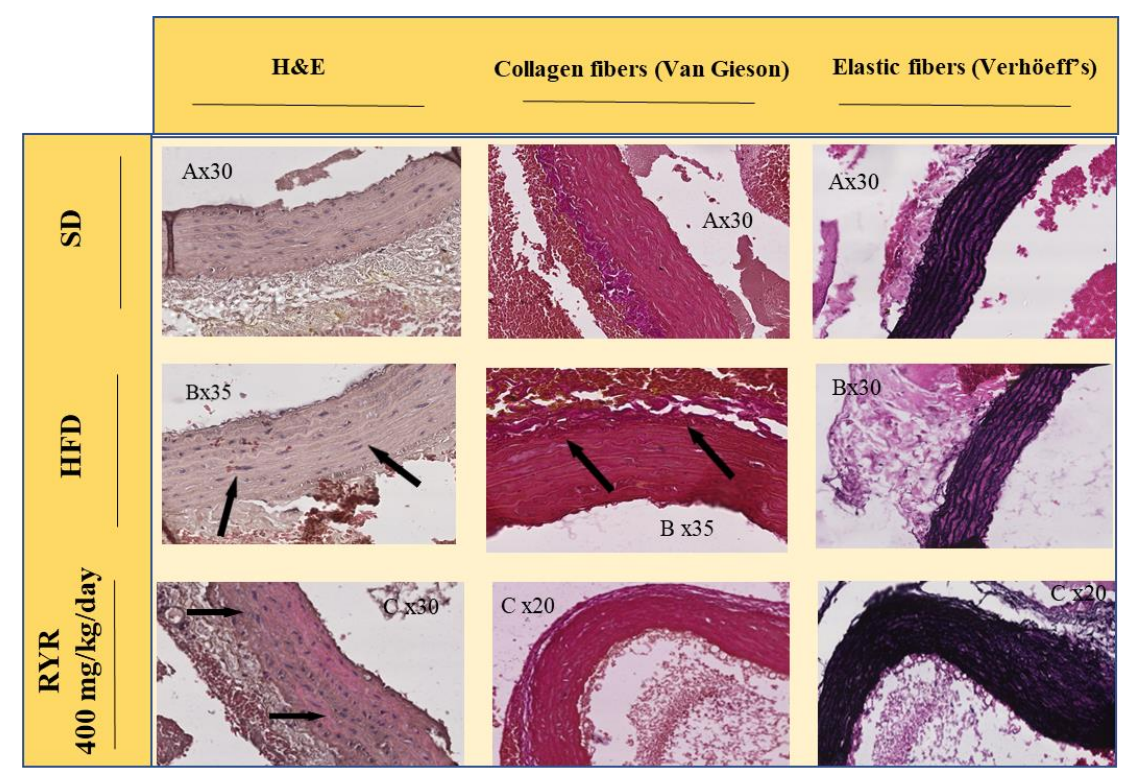

Figure 8. Effect of RYR on histology of isolated rat aorta. Histological sections of the aortae of all animals were stained with hematoxylin and eosin (H \& E) stains to study general structure. Whereas to investigate, collagen and elastic fibers sections were stained with van Gieson and Verhöeff's stains, respectively (x 30).

\section{Results and Discussion}

The vascular endothelium is an active organ that is regulating normal vascular tone and maintaining vascular homeostasis. The role of endothelial dysfunction in the development of atherosclerosis and plaque progression is well established [36]. Endothelial dysfunction ends up with reduced vasodilatation, specifically nitric oxide and/or an increase in endotheliumderived contracting factors [37]. A difference between vasodilation and vasoconstriction leads to functional characteristics of endothelial dysfunction. Pathophysiology of endothelial dysfunction includes multiple cardiovascular risk factors such as oxidative stress, hypercholesterolemia, hypertension, hyperglycemia, aging, and smoking [38,39]. It is wellestablished that obesity is strongly correlated with an elevated risk of cardiovascular, hypertension, and diabetes complications. However, there are limited medicinal treatment agents that can interfere with obesity-related complications, specifically cardiovascular complications. This research was planned to explore the possible positive effects of red yeast extract (RYR) in the major complications of obesity, specifically vascular endothelial dysfunction and dyslipidemia. Based on several studies and experiments, we used two doses of RYR that achieved the best results in our experimental settings, 200 and $400 \mathrm{mg} / \mathrm{kg} / \mathrm{day}$. These doses were used in rats receiving HFD (which induced obesity-related complications like dyslipidemia and vascular endothelial dysfunction) [40].

The first step was to investigate in-vivo model validity. Based on the HFD animals' bodyweight compared to the SD animals, our data demonstrated a significant variance in adiposity between the SD and HFD groups (Figure 2). To further investigate model validity, LW to BW, RFPW to BW, waist, Lee index, and BMI were evaluated in all animals feeding patterns with or without RYR treatment.

As expected, animals that received HFD showed a significant increase in all parameters, suggesting that these animals showed classical signs of obesity compared to those that received SD. These findings are consistent with similar findings previously reported in the literature 
using animals receiving the same formula of HFD [31]. On the other hand, significant reductions were observed in BW, LW/BW, RFPW/BW, waist, Lee index, and BMI in the animals that received RYR treatment of 200 or $400 \mathrm{mg} / \mathrm{kg} /$ day along with the HFD. There was no statistical difference between the effects of the two concentrations in all parameters. These results suggest that RYR had a positive protective effect against obesity, which in turn suggested having a role in lowering obesity-related complications such as dyslipidemia, diabetes, and hypertension.

The feeding pattern and amount of ingestion of all groups during treatment weeks were monitored and showed a consistent food amount. This suggests that bulging and overeating did not have any effect on the obesity-related results (Figure 5). These numerical results are consistent with the rats' visual examination upon an exploration of retroperitoneal fat of various treated animal groups (Figure 6). Animals with SD have shown the least amount of fat and a minor increase in the fat in animals treated with 200 or $400 \mathrm{mg} / \mathrm{kg} /$ day along with the HFD. Finally, HFD animals exhibited the highest fat content, which represents a clear sign of obesity. It is worth mentioning that the selection of the animals for the exploration was made randomly.

It is very well known that HFD can produce significant negative effects on the lipid panel [41]. These effects are suggested to be mediated through increased lipids absorption from the gastrointestinal tract (GIT) and a reduction of cholesterol metabolism. Animals that received HFD have shown several obesity-related dyslipidemia characteristics, such as a marked elevation in serum TC, TG, VLDL, and LDL levels. On the contrary, HFD-fed animals have been shown significantly lower HDL levels as compared to the SD animals. Lipid profile from animals with SD was considered the baseline values.

Based on our results, animals treated with $400 \mathrm{mg} / \mathrm{kg} / \mathrm{day}$ RYR and HFD have shown significantly lower levels of TC, TG, VLDL, and LDL, along with a significant increase in HDL level as compared to the HFD group. However, these values were also statistically different from baseline levels, suggesting partial positive effects on the lipid profile when using the $400 \mathrm{mg}$ of RYR. Moreover, $200 \mathrm{mg}$ RYR treatment has shown a limited positive effect since it did not induce a significant change in TC, HDL, and LDL levels. These results suggest that RYR can significantly improve the lipid profile in HFD treated animals.

Another aspect that has been investigated is the relationship between obesity due to HFD and the vascular endothelium, specifically, the aortic vascular endothelial dysfunction. This parameter is essential since it is highly associated with the excessive deposition of adipose tissue, which in turn affects vascularity since the major function of the endothelium is to form the interface between the circulating blood and the vascular wall.

Literature has clearly shown an association between the increase in blood cholesterol levels with atherosclerotic and angiographically smooth coronary arteries relaxation [42], which is mainly dependent on the vascular endothelium. Based on that, we have selected one of the vasoactive substances, acetylcholine, which regulates systemic vascular resistance.

Our findings suggest that animals treated with RYR for $400 \mathrm{mg} / \mathrm{kg} / \mathrm{day}$ and the HFD did not show the endothelial dysfunction signs, which was very clear in the HFD-animals. This is represented by the significant increase in acetylcholine-induced vasorelaxation in the animals that received RYR as compared to the HFD animals. The levels of protection against endothelial dysfunction were great at the $400 \mathrm{mg} / \mathrm{kg} /$ day and very close to the SD animals' baseline values. However, the $200 \mathrm{mg} / \mathrm{kg} /$ day provided partial protection, which suggests a dose-dependent vascular endothelial protection. 
Moreover, the aorta's vascular histology in all categories was investigated since it is highly suggested that obesity can induce physical histological changes in vascular endothelium tissues [43]. Our results suggest that the aortic tissues, which are typical vascular endothelium tissues of the HFD animals, have shown fat accumulation signs. These signs include an increase in adipocytes, a reduction in the elastic fiber layers, and an increase in the collagen-elastic ratio demonstrated by an increase in collagen layer compared to aortic tissues of the SD animals. These findings are associated with many reports in the literature that linked the increase of the body mass index (BMI) to reduce aortic tissue elasticity, which is an important indicator of the cardiovascular complication of obesity [44]. However, pharmacological intervention, specifically when using $400 \mathrm{mg} / \mathrm{kg} / \mathrm{day}$, resulted in significant protection against the complication caused by HFD-induced obesity, such as aortic stiffness. This resulted in an overall lowering of endothelium degradation signs, such as collagen-elastic ratio.

\section{Conclusions}

The present study results conclude the antidyslipidemic and endothelial protective effects of RYR as evident by improved lipid profile and vascular endothelial function. Hence, it justifies the therapeutic role of RYR in the treatment of hyperlipidemia and related cardiovascular conditions. However, more systematic studies are still needed to isolate and identify pure active compounds of RYR to confirm their beneficial role.

\section{Funding}

This research received no external funding.

\section{Acknowledgments}

The authors are thankful to the Deanship of Scientific Research (DSR), Prince Sattam bin Abdulaziz University, Al-Kharj, Kingdom of Saudi Arabia, for supporting the study.

\section{Conflicts of Interest}

The authors declare no conflict of interest.

\section{References}

1. De Lorenzo, A.; Gratteri, S.; Gualtieri, P.; Cammarano, A.; Bertucci, P.; Di Renzo, L. Why primary obesity is a disease? J. Transl. Med. 2019, 17, 169, https://doi.org/10.1186/s12967-019-1919-y.

2. Chiurazzi, M.; Cozzolino, M.; Orsini, R.C.; Di Maro, M.; Di Minno, M.N.; Colantuoni, A. Impact of Genetic Variations and Epigenetic Mechanisms on the Risk of Obesity. Int. J. Mol. Sci. 2020, 21, https://doi.org/10.3390/ijms21239035.

3. Clément, K.; Vaisse, C.; Manning, B.S.J.; Basdevant, A.; Guy-Grand, B.; Ruiz, J.; Silver, K.D.; Shuldiner, A.R.; Froguel, P.; Strosberg, A.D. Genetic variation in the $\beta 3$-adrenergic receptor and an increased capacity to gain weight in patients with morbid obesity. New Engl. J. Med. 1995, 333, 352-354, https://doi.org/10.1056/NEJM199508103330605.

4. Ristow, M.; Müller-Wieland, D.; Pfeiffer, A.; Krone, W.; Kahn, C.R. Obesity Associated with a Mutation in a Genetic Regulator of Adipocyte Differentiation. New Engl. J. Med. 1998, 339, 953-959, https://doi.org/10.1056/NEJM199810013391403.

5. Voinea, L.; Popescu, D.V.; Bucur, M.; Negrea, T.M.; Dina, R.; Enache, C. Reshaping the Traditional Pattern of Food Consumption in Romania through the Integration of Sustainable Diet Principles. A Qualitative Study. Sustainability 2020, 12, https://doi.org/10.3390/su12145826. 
6. Friedman, J.M. A War on Obesity, Not the Obese. Science 2003, 299, 856, https://doi.org/10.1126/science.1079856.

7. Hill, J.O.; Wyatt, H.R.; Reed, G.W.; Peters, J.C. Obesity and the Environment: Where Do We Go from Here? Science 2003, 299, 853, https://doi.org/10.1126/science.1079857.

8. Ss, M.A. A review of prevalence of obesity in Saudi Arabia. J Obes Eat Disord 2016, 2, 1-6, https://doi.org/10.21767/2471-8203.100025.

9. Ogden, C.L.; Carroll, M.D.; Flegal, K.M. Epidemiologic trends in overweight and obesity. Endocrinol. Metab. Clin. North Am. 2003, 32, 741-760, vii, https://doi.org/10.1016/s0889-8529(03)00074-4.

10. Ogden, C.L.; Carroll, M.D.; Curtin, L.R.; McDowell, M.A.; Tabak, C.J.; Flegal, K.M. Prevalence of Overweight and Obesity in the United States, 1999-2004. JAMA 2006, 295, 1549-1555, https://doi.org/10.1001/jama.295.13.1549.

11. Al-Qahtani, A.M. Prevalence and Predictors of Obesity and Overweight among Adults Visiting Primary Care Settings in the Southwestern Region, Saudi Arabia. BioMed Research International 2019, 2019, 8073057, https://doi.org/10.1155/2019/8073057.

12. Ait-Aissa, K.; Nguyen, Q.M.; Gabani, M.; Kassan, A.; Kumar, S.; Choi, S.-K.; Gonzalez, A.A.; Khataei, T.; Sahyoun, A.M.; Chen, C.; Kassan, M. MicroRNAs and obesity-induced endothelial dysfunction: key paradigms in molecular therapy. Cardiovasc. Diabetol. 2020, 19, 136, https://doi.org/10.1186/s12933-02001107-3.

13. Vanhoutte, P.M.; Shimokawa, H.; Tang, E.H.C.; Feletou, M. Endothelial dysfunction and vascular disease. Acta Physiologica 2009, 196, 193-222, https://doi.org/10.1111/j.1748-1716.2009.01964.x.

14. Ozdemir, B.; Yazici, A. Could the decrease in the endothelial nitric oxide (NO) production and NO bioavailability be the crucial cause of COVID-19 related deaths? Med. Hypotheses 2020, 144, 109970, https://doi.org/10.1016/j.mehy.2020.109970.

15. Gamrat, A.; Surdacki, M.A.; Chyrchel, B.; Surdacki, A. Endothelial Dysfunction: A Contributor to Adverse Cardiovascular Remodeling and Heart Failure Development in Type 2 Diabetes beyond Accelerated Atherogenesis. Journal of Clinical Medicine 2020, 9, https://doi.org/10.3390/jcm9072090.

16. Lasker, S.; Rahman, M.M.; Parvez, F.; Zamila, M.; Miah, P.; Nahar, K.; Kabir, F.; Sharmin, S.B.; Subhan, N.; Ahsan, G.U.; Alam, M.A. High-fat diet-induced metabolic syndrome and oxidative stress in obese rats are ameliorated by yogurt supplementation. Sci. Rep. 2019, 9, 20026, https://doi.org/10.1038/s41598-01956538-0.

17. onda, P.Y.; Poondla, V.; Jaiswal, K.K.; Dasari, S.; Uyyala, R.; Surtineni, V.P.; Egi, J.Y.; Masilamani, A.J.A.; Bestha, L.; Konanki, S.; Muthulingam, M.; Lingamgunta, L.K.; Aloor, B.P.; Tirumalaraju, S.; Sade, A.; Ratnam Kamsala, V.; Nagaraja, S.; Ramakrishnan, R.; Natesan, V. Pathophysiology of high fat diet induced obesity: impact of probiotic banana juice on obesity associated complications and hepatosteatosis. Sci. Rep. 2020, 10, 16894, https://doi.org/10.1038/s41598-020-73670-4.

18. Labban, R.S.M.; Alfawaz, H.; Almnaizel, A.T.; Hassan, W.M.; Bhat, R.S.; Moubayed, N.M.S.; Bjørklund, G.; El-Ansary, A. High-fat diet-induced obesity and impairment of brain neurotransmitter pool. Transl. Neurosci. 2020, 11, 147-160, https://doi.org/10.1515/tnsci-2020-0099.

19. AlAmri, O.D.; Albeltagy, R.S.; M. A. Akabawy, A.; Mahgoub, S.; Abdel-Mohsen, D.M.; Abdel Moneim, A.E.; Amin, H.K. Investigation of antioxidant and anti-inflammatory activities as well as the renal protective potential of green coffee extract in high fat-diet/streptozotocin-induced diabetes in male albino rats. J. Funct. Foods 2020, 71, 103996, https://doi.org/10.1016/j.jff.2020.103996.

20. Veeresham, C. Natural products derived from plants as a source of drugs. J. Adv. Pharm. Technol. Res. 2012, 3, 200, https://doi.org/10.4103/2231-4040.104709.

21. Boccellino, M.; D’Angelo, S. Anti-Obesity Effects of Polyphenol Intake: Current Status and Future Possibilities. Int. J. Mol. Sci. 2020, 21, https://doi.org/10.3390/ijms21165642.

22. Abdul Rahman, H.; Saari, N.; Abas, F.; Ismail, A.; Mumtaz, M.W.; Abdul Hamid, A. Anti-obesity and antioxidant activities of selected medicinal plants and phytochemical profiling of bioactive compounds. Int. J. Food Prop. 2017, 20, 2616-2629, https://doi.org/10.1080/10942912.2016.1247098.

23. Chairote, E.; Chairote, G.; Wongpornchai, S.; Lumyong, S. Preparation of red yeast rice using various Thai glutinous rice and Monascus purpureus CMU001 isolated from commercial Chinese red yeast rice sample. CURRENT APPLIED SCIENCE AND TECHNOLOGY 2007, 7, 28-37.

24. Liu, J.; Zhang, J.; Shi, Y.; Grimsgaard, S.; Alraek, T.; Fønnebø, V. Chinese red yeast rice (Monascus purpureus) for primary hyperlipidemia: a meta-analysis of randomized controlled trials. Chin. Med. 2006, 1, 4, https://doi.org/10.1186/1749-8546-1-4 
25. Li, Y.; Jiang, L.; Jia, Z.; Xin, W.; Yang, S.; Yang, Q.; Wang, L. A Meta-Analysis of Red Yeast Rice: An Effective and Relatively Safe Alternative Approach for Dyslipidemia. PLoS One 2014, 9, e98611, https://doi.org/10.1371/journal.pone.0098611.

26. Scicchitano, P.; Cameli, M.; Maiello, M.; Modesti, P.A.; Muiesan, M.L.; Novo, S.; Palmiero, P.; Saba, P.S.; Pedrinelli, R.; Ciccone, M.M. Nutraceuticals and dyslipidaemia: Beyond the common therapeutics. J. Funct. Foods 2014, 6, 11-32, https://doi.org/10.1016/j.jff.2013.12.006.

27. Gerards, M.C.; Terlou, R.J.; Yu, H.; Koks, C.H.W.; Gerdes, V.E.A. Traditional Chinese lipid-lowering agent red yeast rice results in significant LDL reduction but safety is uncertain-a systematic review and metaanalysis. Atherosclerosis 2015, 240, 415-423, https://doi.org/10.1016/j.atherosclerosis.2015.04.004.

28. Visioli, F.; Poli, A. Prevention and Treatment of Atherosclerosis: The Use of Nutraceuticals and Functional Foods. Springer Berlin Heidelberg: Berlin, Heidelberg, https://doi.org/10.1007/164_2019_341.

29. Zhang, Z.; Ali, Z.; Khan, S.I.; Khan, I.A. Cytotoxic monacolins from red yeast rice, a Chinese medicine and food. Food Chem. 2016, 202, 262-268, https://doi.org/10.1016/j.foodchem.2015.12.039.

30. Frishman, W.H.; Rapier, R.C. Lovastatin: an HMG-CoA reductase inhibitor for lowering cholesterol. Med. Clin. North Am. 1989, 73, 437-448, https://doi.org/10.1016/s0025-7125(16)30681-2.

31. Xu, S.-P.; Mao, X.-Y.; Cheng, X.; Chen, B. Ameliorating effects of casein glycomacropeptide on obesity induced by high-fat diet in male Sprague-Dawley rats. Food Chem. Toxicol. 2013, 56, 1-7, https://doi.org/10.1016/j.fct.2013.01.027.

32. Diniz, Y.S.; Faine, L.A.; Galhardi, C.M.; Rodrigues, H.G.; Ebaid, G.X.; Burneiko, R.C.; Cicogna, A.C.; Novelli, E.L.B. Monosodium glutamate in standard and high-fiber diets: metabolic syndrome and oxidative stress in rats. Nutrition 2005, 21, 749-755, https://doi.org/10.1016/j.nut.2004.10.013.

33. Bernardis, L.L. Prediction of carcass fat, water and lean body mass from Lee's 'nutritive ratio' in rats with hypothalamic obesity. Experientia 1970, 26, 789-790, https://doi.org/10.1007/BF02232553.

34. Madkhali, H.A. Morin attenuates high-fat diet induced-obesity related vascular endothelial dysfunction in Wistar albino rats. Saudi Pharmaceutical Journal 2020, 28, 300307, https://doi.org/10.1016/j.jsps.2020.01.009.

35. Furchgott, R.F.; Zawadzki, J.V. The obligatory role of endothelial cells in the relaxation of arterial smooth muscle by acetylcholine. Nature 1980, 288, 373-376, https://doi.org/10.1038/288373a0.

36. Kinlay, S.; Ganz, P. Role of endothelial dysfunction in coronary artery disease and implications for therapy. Am. J. Cardiol. 1997, 80, 11i-16i, https://doi.org/10.1016/s0002-9149(97)00793-5.

37. Lerman, A.; Burnett, J.C., Jr. Intact and altered endothelium in regulation of vasomotion. Circulation 1992, 86, Iii12-19.

38. Libby, P.; Ridker Paul, M.; Maseri, A. Inflammation and Atherosclerosis. Circulation 2002, 105, 1135-1143, https://doi.org/10.1161/hc0902.104353.

39. Paravicini, T.M.; Touyz, R.M. NADPH Oxidases, Reactive Oxygen Species, and Hypertension. Diabetes Care 2008, 31, S170, https://doi.org/10.2337/dc08-s247.

40. Xie, X.; Wang, Y.; Zhang, S.; Zhang, G.; Xu, Y.; Bi, H.; Daugherty, A.; Wang, J.-a. Chinese red yeast rice attenuates the development of angiotensin II-induced abdominal aortic aneurysm and atherosclerosis. J. Nutr. Biochem. 2012, 23, 549-556, https://doi.org/10.1016/j.jnutbio.2011.02.011.

41. Al-Dosari, M.; Alqasoumi, S.; Ahmed, M.; Al-Yahya, M.; Ansari, M.N.; Rafatullah, S. Effect of Beta vulgaris L. on cholesterol rich diet-induced hypercholesterolemia in rats. Farmacia 2011, 59, 669-678.

42. Vancheri, F.; Longo, G.; Vancheri, S.; Henein, M. Coronary Microvascular Dysfunction. Journal of Clinical Medicine 2020, 9, https://doi.org/10.3390/jcm9092880.

43. Esper, R.J.; Machado, R.; Vilariño, J.; Cacharrón, J.L.; Ingino, C.A.; García Guiñazú, C.A.; Bereziuk, E.; Bolaño, A.L.; Suarez, D.H. Endothelium-dependent responses in patients with hypercholesterolemic coronary artery disease under the effects of simvastatin and enalapril, either separately or combined. Am. Heart J. 2000, 140, 684-689, https://doi.org/10.1067/mhj.2000.109649.

44. Tryggestad, J.B.; Thompson, D.M.; Copeland, K.C.; Short, K.R. Obese children have higher arterial elasticity without a difference in endothelial function: the role of body composition. Obesity (Silver Spring, Md.) 2012, 20, 165-171, https://doi.org/10.1038/oby.2011.309. 